\title{
Angiographic and Pathologic Features of Probable Primary Carcinoid-like Hepatic Tumors
}

\author{
Joseph F. Walter, ${ }^{1}$ Henry D. Appelman, and Stewart R. Reuter ${ }^{2}$ \\ Department of Radiology and Pathology, University of Michigan, Ann Arbor, Michigan, USA
}

\begin{abstract}
The angiographic and pathologic features of two cases of primary carcinoid-like hepatic tumors are described. Neither patient had the carcinoid syndrome.
\end{abstract}

Key words: Abdominal angiography - Liver, primary tumor - Liver, carcinoid.

In his classic monograph, Tumor of the Liver and Intrahepatic Bile Ducts, Edmondson [1] refers anecdotally to 2 patients with carcinoid-like tumors of the liver in whom careful autopsies failed to reveal a primary neoplasm. He therefore considered them to be true primary hepatic neoplasms. This concept was reinforced by Alpert et al. [2], who described cholangiocarcinomas with carcinoidal components. The current report describes angiographic and pathologic features of 2 patients with proven primary carcinoid-like liver tumors without the carcinoid syndrome.

\section{Case Reports}

Case 1. A 52-year-old woman had upper abdomen and back pain. An upper GI series demonstrated a scarred duodenum and she underwent a subtotal gastrectomy and gastrojejunostomy. A mass was discovered incidentally deep in the left lobe of the liver and biopsied. The histologic diagnosis interpreted variously to be metastatic adenocarcinoma, primary hepatoma, or metastatic islet cell tumor. The only biochemical abnormality was a slight elevation of alkaline phosphatase. No symptoms suggested hormonal function.

She was referred to the University of Michigan Medical Center

1 Department of Radiology, Palo Alto Medical Clinic, 300 Homer Avenue, Palo Alto, CA 94301, USA; and ${ }^{2}$ Department of Radiology, Martinez V.A. Hospital, Martinez, CA 94553, USA.

Address reprint requests to: J.F. Walter, M.D., Department of Radiology, Palo Alto Medical Clinic, 300 Homer Avenue, Palo Alto, CA 94301, USA.
(UMMC) where angiography demonstrated a large neoplasm involving most of the left lobe of the liver with extension into the right lobe (Fig. 1). The major feeding vessel, the left hepatic artery, was dilated. There was scant fine tumor neovasculature, but the predominant angiographic feature was an intense homogeneous vascular tumor blush. No arteriovenous shunts or vascular lakes were present. The portal veins were normal but the hepatic veins were not studied. There was no angiographic evidence of a primary lesion in the pancreas or intestine.

At operation no extrahepatic primary neoplasm was found despite extensive exploration of the intestine and pancreas, and a left hepatic resection was done. She required reexploration because of postoperative hemorrhage and died. Unfortunately, no autopsy was done.

The large hepatic tumor was partly encapsulated. It was composed of slightly pleomorphic epithelial cells with eccentric nuclei and pale cytoplasm. The cells were arranged in cords and nests, separated by fine fibrovascular septa. Occasional tumor cells contained argyrophillic granules. This general pattern is common to a group of ncuroendocrinc tumors which include carcinoid and islet cell tumors (Fig. 2). Unfortunately, no electron microscopic evaluation was done.

Case 2. A 55 year-old man had undergone abdominal exploration for pain 2 years previously at which time a tumor was found incidentally in the left lobe of the liver. The biopsy was originally interpreted as hepatoma, although the diagnosis was later changed to metastatic carcinoid. He had no symptoms of the carcinoid syndrome. For 2 years he was treated with intravenous 5-fluorouracil and manifested no clinical progression of the tumor.

$\mathrm{He}$ was referred to the UMMC where angiography demonstrated an extensive hypervascular tumor in the left lobe of the liver (Fig. 3). The hepatic artery was dilated and numerous abnormal arteries supplied the mass. An extensive tumor stain was present in the capillary phase which was less homogeneous than in case 1. The mass was solitary with the exception of a separate $2-\mathrm{cm}$ nodule in the right lobc. The portal vein was smoothly compressed by tumor extending toward the hilum of the liver. No arteriovenous shunting, lakes of contrast accumulation, or venous invasion were demonstrated. The hepatic veins were not studied. No primary lesions were found in the pancreas or intestine despite use of superselective magnification angiography.

At exploration the tumor was considered to be unresectable because the smaller nodule demonstrated at angiography was reported to be "metastatic carcinoma" on frozen section. The portal vein was explored with the intent of ligation, but it was severely compressed by tumor and this was not possible. The 

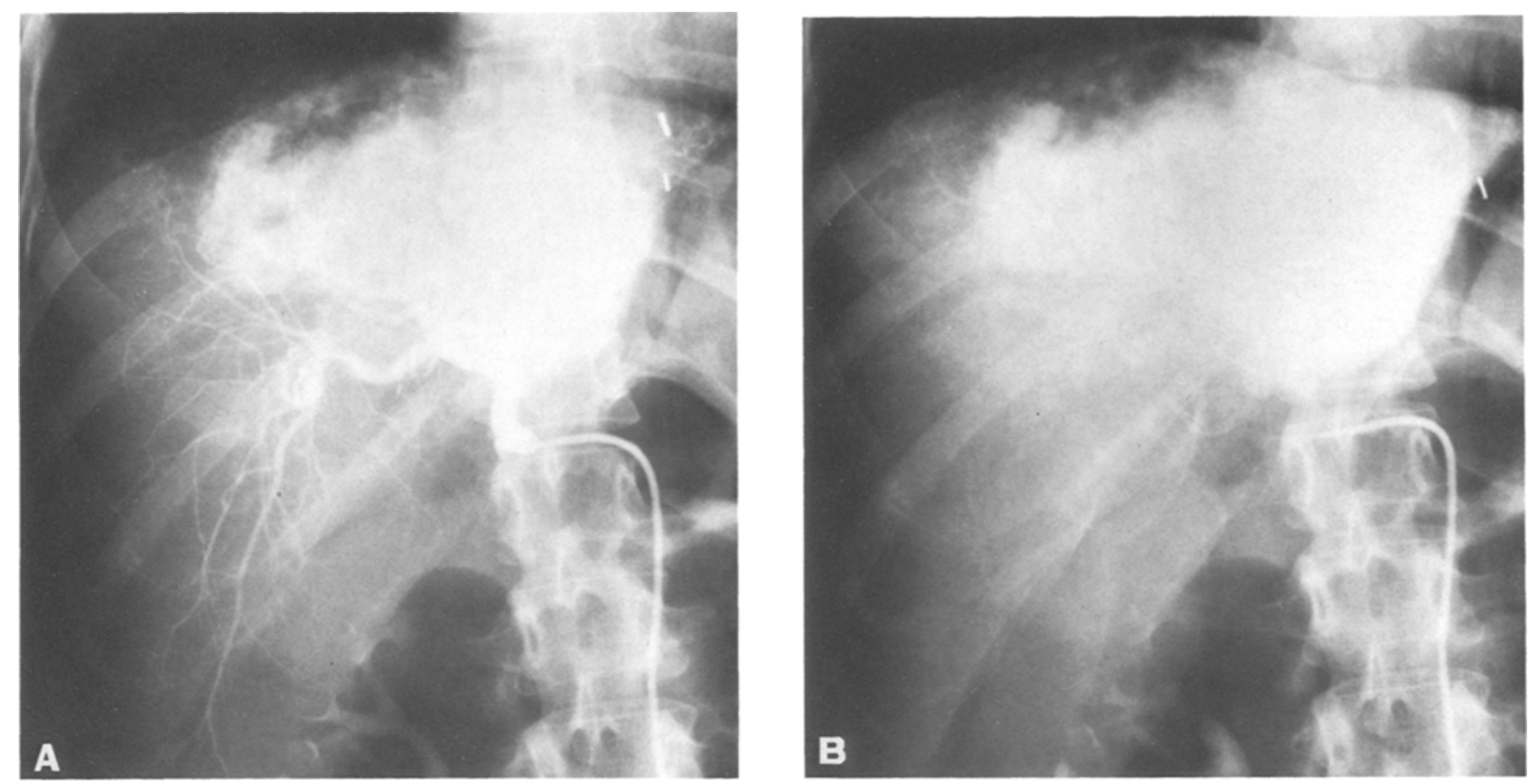

Fig. 1. Case I. A The arterial phase of a selective hepatic angiogram demonstrates a dilated left hepatic artery and scant neovasculature in solitary left lobe mass. Note the absence of arteriovenous shunting or contrast lakes B The capillary phase demonstrates a dense, homogeneous hypervascular mass occupying the entire left lobe. The portal vein had been normal on a previous celiac injection. Surgical clips remain from a previous vagotomy
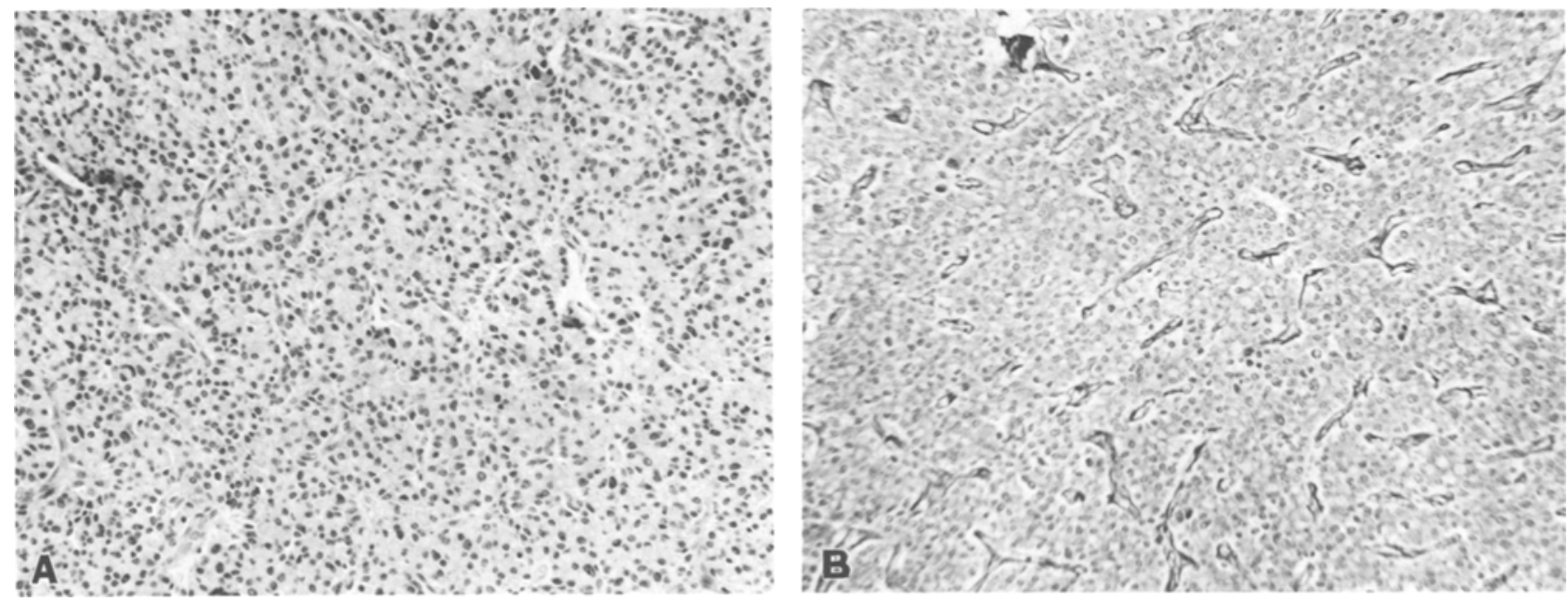

Fig. 2. Representative sections of case 1. A In most areas there were cords and nests of generally uniform epithelial cells surrounded by small vessels $(H \& E ; \times 163)$. B Reticulin stain emphasizes the diffusely distributed small vessels which explain the dense angiographic tumor blush. Both tumors were histologically similar except for coarser septation in case 2

pancreas and entire GI tract were palpated and no primary neoplasms were found.

This tumor was histologically similar to that in case 1 , but in addition, the strands of cells often surrounded vascular lumina. Aiso, the septa were more thickly collagenized, separating the tumor into large nodules. No intracytoplasmic granules were found by either argyrophillic or argentaffin staining techniques.

The patient has been treated with hepatic artery and intravenous infusion of 5-fluorouracil and has not deteriorated in the
4 years since his initial diagnosis. The prolonged course suggests that these tumors may grow slowly.

\section{Discussion}

Carcinoid-like tumors of the liver and biliary tract are extremely rare and have usually been reported 

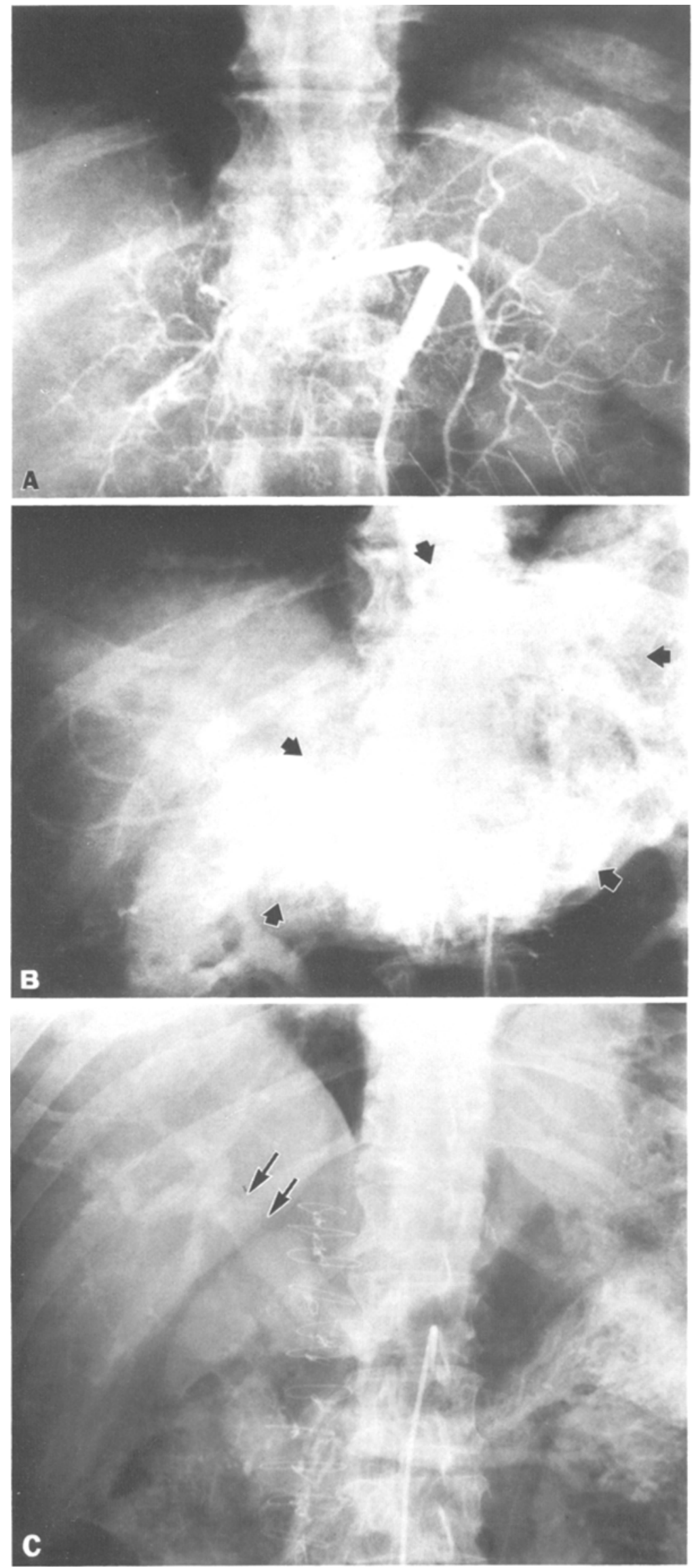

Fig. 3. Case 2. A The arterial phase of a selective left gastric angiogram demonstrates a dilated left hepatic artery and scant neovasculature.

B The capillary phase demonstrates the densely staining tumor expanding the left lobe. No shunting or laking evident.
C The venous phase of a celiac angiogram demonstrates an impression on the portal vein in the liver hilum. At operation the portal vein was compressed but not invaded 
as isolated cases [3-5]. Only 2 cases occurred among 2,837 carcinoid tumors reviewed by Godwin [6]. Most occur in the gallbladder, but the presumed precursor cell, the Kulchitsky cell, occurs throughout the gastrointestinal tract with the possible exception of the esophagus.

The angiographic abnormalities caused by these two liver neoplasms are distinctive. Both tumors had a rather homogeneous, "cloud-like" tumor stain in the capillary phase, similar to that of most islet cell adenomas of the pancreas. This finding corresponds well to the uniform capillary pattern of the tumors seen histologically. Case 1 was homogeneous at angiography and histologically had very fine septa. Case 2 had a less homogeneous angiographic appearance corresponding well with its much thicker collagenized septation.

The supplying hepatic artery branches were slightly tortuous, but no wild neovasculature, arteriovenous shunts, or vascular lakes, which typify most hepatocellular carcinomas, were present [7]. Arterial encasement, the primary feature of the usual primary cholangiocarcinoma, was also absent [8]. Although the portal veins were not invaded, external compression of the portal vein was confirmed at exploration in one of the patients.

To out knowledge, the detailed angiographic findings of primary hepatic carcinoid-like tumors have not been described previously. Alpert et al. [2] refer to a "vascular tumor blush" in their case. Goldstein and Miller [9] illustrate similar features in hepatic metastases from carcinoid tumors of the small intestine. Except for the fact that hepatic metastases from carcinoid tumors are almost always multiple, the angiographic features, likely the result of the diffusely distributed capillaries surrounding small nests of tumor cells, are similar to those of the cases reported here.

\section{References}

1. Edmondson, HA: Tumors of the liver and intrahepatic bile ducts. In: Atlas of Tumor Pathology, Armed Forces Institute of Pathology, 1958

2. Alpert LI, Zak FG, Werthamer S, Bochetto JF: Cholangiocarcinoma. A clinicopathologic study of five cases with ultrastructural observation. Hum Pathol 5: 709-728, 1974

3. Shiffman MA, Juler G: Carcinoid of the biliary tract. Arch Surg 89:1113-1115, 1964

4. Barnes TG: Argentaffinoma (carcinoid) of gallbladder. Surgery 32:723-727, 1952

5. Bosse MD: Carcinoid tumor of gallbladder. Arch Pathol $35: 898-899,1943$

6. Godwin JD: Carcinoid tumors. An analysis of 2,837 cases. Cancer 36:560-569, 1975

7. Reuter SR, Redman HC, Siders DB: The spectrum of angiographic findings in hepatoma. Radiology 94:89-94, 1970

8. Walter JF, Bookstein JJ, Bouffard EV: Newer angiographic observations in cholangiocarcinoma. Radiology 118:19-23, 1976

9. Goldstein HM, Miller M: Angiographic evaluation of carcinoid tumor of the small intestine: the value of epinephrine. Radiology $115: 23-28,1975$

Received: March 3, 1978; accepted after revision: May 3, 1978 